\title{
CONSIDERACIÓN DE LA AMENAZA SÍSMICA EN EL ORDENAMIENTO TERRITORIAL DEL CANTÓN DE POÁS, COSTA RICA
}

\author{
Gustavo Barrantes-Castillo'; Elkin de Jesús Salcedo-Hurtado²
}

DOI: http://dx.doi.org/10.18273/revbol.v38n3-2016007 (c) (i) త

Forma de citar: Barrantes, G., y Salcedo, E. 2016. Consideración de la amenaza sísmica en el Ordenamiento Territorial del cantón de Poás, Costa Rica. Boletín de Geología, 38(3): 109-127.

\section{RESUMEN}

La valoración de la amenaza sísmica para su incorporación en el Ordenamiento Territorial puede ser realizada por medio de la combinación de los métodos propios de la sismología y del uso de Sistemas de Información Geográfica (SIG), evitando el uso de simplificaciones que son impulsadas en la normativa costarricense. En esta investigación se ejemplifica la aplicación del método determinístico en la evaluación de la amenaza símica del cantón de Poás, provincia de Alajuela, Costa Rica. A partir de este enfoque se identificaron las fuentes sísmicas que representan la mayor amenaza para el cantón y sus magnitudes máximas posibles, la atenuación sísmica, así como los valores de aceleración (PGA) esperados que son trasformados en intensidades (IMM); con base en lo cual se eligió el peor escenario posible. El uso de un SIG facilitó la implementación de las fórmulas a nivel de píxeles de $5 \times 5 \mathrm{~m}$, lo que hizo posible la elaboración de cartografía de amenaza a una escala apropiada para su implementación en el Ordenamiento Territorial. Los resultados obtenidos muestran que la parte norte del cantón presenta la mayor amenaza (IMM IX), y que la ciudad principal, San Pedro, puede verse afectada por una intensidad máxima de IX en el peor escenario.

Palabras clave: amenaza sísmica, PGA, cantón Poás, Ordenamiento Territorial.

\section{CONSIDERATION OF THE SEISMIC HAZARD ON THE LAND USE PLANNING OF THE POÁS CANTON, COSTA RICA}

\begin{abstract}
To incorporate the seismic hazard assessment in the land use planning can be done using seismic methods and Geographic Information System (GIS), in order to avoid the use of simplifications provided in law. In this paper, we used the deterministic method for assessment of the seismic hazard in Poás County, Province of Alajuela, Costa Rica. Following this method, we identified the seismic sources that represent the main threat to the county, and the possible maximum magnitudes; the seismic attenuation, as well as the maximum acceleration values that were transformed into seismic intensity. The use of a GIS allowed the estimate of values in a $5 \times 5 \mathrm{~m}$ mesh, in order to generate the seismic hazard maps at appropriate scale for the land use planning. The results have shown that the northern part of the County has a high seismic hazard (IMM IX) as well as San Pedro town, according to the worst scenario possible.
\end{abstract}

Keywords: seismic hazard assessment, PGA, Poás County, land use planning.

\footnotetext{
${ }^{1}$ Escuela de Ciencias Geográficas, Universidad Nacional, Costa Rica, gbarrantes@una.cr

${ }^{2}$ Grupo Georiesgos del Observatorio Sismológico y Geofísico del Suroccidente Colombiano, Departamento de Geografía, Universidad del Valle. Ciudad universitaria. Cali-Colombia. A. A. 25360, elkin.salcedo@correounivalle.edu.co
} 


\section{INTRODUCCIÓN}

En Costa Rica, la incorporación de las amenazas naturales en el Ordenamiento Territorial (OT) se realiza por medio del "Índice de Fragilidad Ambiental" (IFA), metodología establecida en el Decreto $\mathrm{N}^{\circ}$ 32967-MINAE (Poder ejecutivo, 2006). Obviando el hecho de que el IFA realiza una inadecuada valoración de las amenazas naturales en términos generales, como lo expusiera Barrantes (2012) en su revisión de dicha metodología, el método propuesto para el análisis de la sismicidad, en un contexto territorial, lleva a simplificaciones no sustentadas en los métodos de evaluación de la amenaza sísmica. En el decreto se establece la valoración de la amenaza sísmica local (para cada municipio) por medio del "índice de densidad sísmica" valor que corresponde con la densidad promedio de sismos por kilómetro cuadrado considerando la magnitud promedio de eventos sísmicos (Decreto No 32967-MINAE).

Si bien el espíritu de esta normativa es el de facilitar una aproximación heurísticas de bajo costo al problema de la valoración de la amenaza sísmica para el OT, con la presente investigación se pretende demostrar que es posible hacer uso de un método de valoración de la amenaza sísmica, como el determinístico, en un contexto territorial. La adaptación realizada se apoya en el empleo de Sistemas de Información Geográfica (SIG) como herramienta para procesar los datos y como medio para el análisis espacial de los resultados de la estimación de la amenaza sísmica.

Como caso de estudio se escogió el cantón de Poás, Costa Rica, municipio que se ubica dentro del área de afectación de varios terremotos, como el de Fraijanes de 1888 y el de Cinchona de 2009. En este cantón, los usos predominantes de la tierra son el agropecuario y de protección natural, no obstante, está experimentando un acelerado crecimiento urbano que tiende a ascender por las laderas del volcán Poás. Actualmente, el municipio está en proceso de elaboración del Plan de Ordenamiento Territorial (llamado Plan Regulador en Costa Rica) por lo que se requieren de estudios de amenaza que puedan complementar los resultados del índice de densidad sísmica que solicita la normativa.

\section{ÁREA DE ESTUDIO}

El área de estudio comprende el cantón de Poás, el cual se ubica en el Valle Central de Costa Rica, América Central. Su extensión máxima es de $21,5 \mathrm{~km}$ desde la cima del Volcán Poás a $2.700 \mathrm{~m}$ de altitud, hasta la confluencia del río Prendas con el río Poás a $650 \mathrm{~m}$ de altitud, en dirección NNE-SSO (FIGURA 1). Se trata de un área elongada de materiales volcánicos disectados por valles en forma de $\mathrm{V}$, que en ocasiones forman pronunciados cañones. Las pendientes más fuertes se encuentran en su parte alta y disminuyen paulatinamente hasta volverse un relieve ondulado, en el extremo sur, rodeado por profundos cañones resultado de la presencia de gruesos mantos de lava.

Poás es el octavo cantón de la provincia de Alajuela. Su extensión es $73,84 \mathrm{~km}^{2}$, y su población es de 29.199 personas según el censo de 2011 (INEC, 2012).

\section{MARCO TEÓRICO}

El Ordenamiento Territorial (OT) puede ser entendido como un proceso integrador de la planificación global y sectorial, tomando como base el territorio, orientado al planeamiento urbano y del uso de la tierra (Massiris, 2005; Pujadas y Font, 1998). Se trata de un proceso de planificación técnico-político que pretende organizar el uso y ocupación del territorio, acorde con las potencialidades y las limitaciones del mismo y de las aspiraciones de su población (Herrera, 2011). La planificación territorial se concreta mediante un plan de OT, que procura ordenar la multiplicidad de usos del suelo que existen o podrían existir en un futuro próximo.

La incorporación de la gestión del riesgo de desastre en el marco del OT puede entenderse como la definición $\mathrm{y}$ adopción de un grupo de medidas regulatorias y programáticas tendientes a reducir el riesgo presente y evitar el riesgo futuro (PREDECAN, 2009). No obstante, los gobiernos locales no tienen competencias para la realización de estudios de amenaza, para definir el tipo y alcance de éstos estudios o criterio técnicos para interpretar los resultados de estudios preexistentes. A pesar de esto, los resultados de la valoración de las amenazas se utilizan para sustentar decisiones de carácter regulatorio o programático, así como en procesos de gestión territorial, tales como el otorgamiento de permisos de construcción y de cambios de uso del suelo (PREDECAN, 2009).

Por estas razones se han desarrollado guías para la incorporación de la gestión del riesgo en el OT, las cuales pretenden ofrecer un procedimiento simple pero de base científico que permita aproximaciones cada vez más detalladas de la amenaza y del riesgo en general. Tal es el caso de los decretos $\mathrm{N}^{\circ}$ 32967-MINAE y $\mathrm{N}^{\circ}$ 1807-MIVIVIENDA en Costa Rica y Colombia respectivamente (Poder ejecutivo, 2006; Ministerio de Vivienda, Ciudad y Territorio, 2014 ) y del manual para la gestión municipal del riesgo de desastres patrocinado por la Comisión Nacional Prevención de Desastres y Atención de Emergencias (CNE) de Costa Rica (Saborío et al., 2014). 


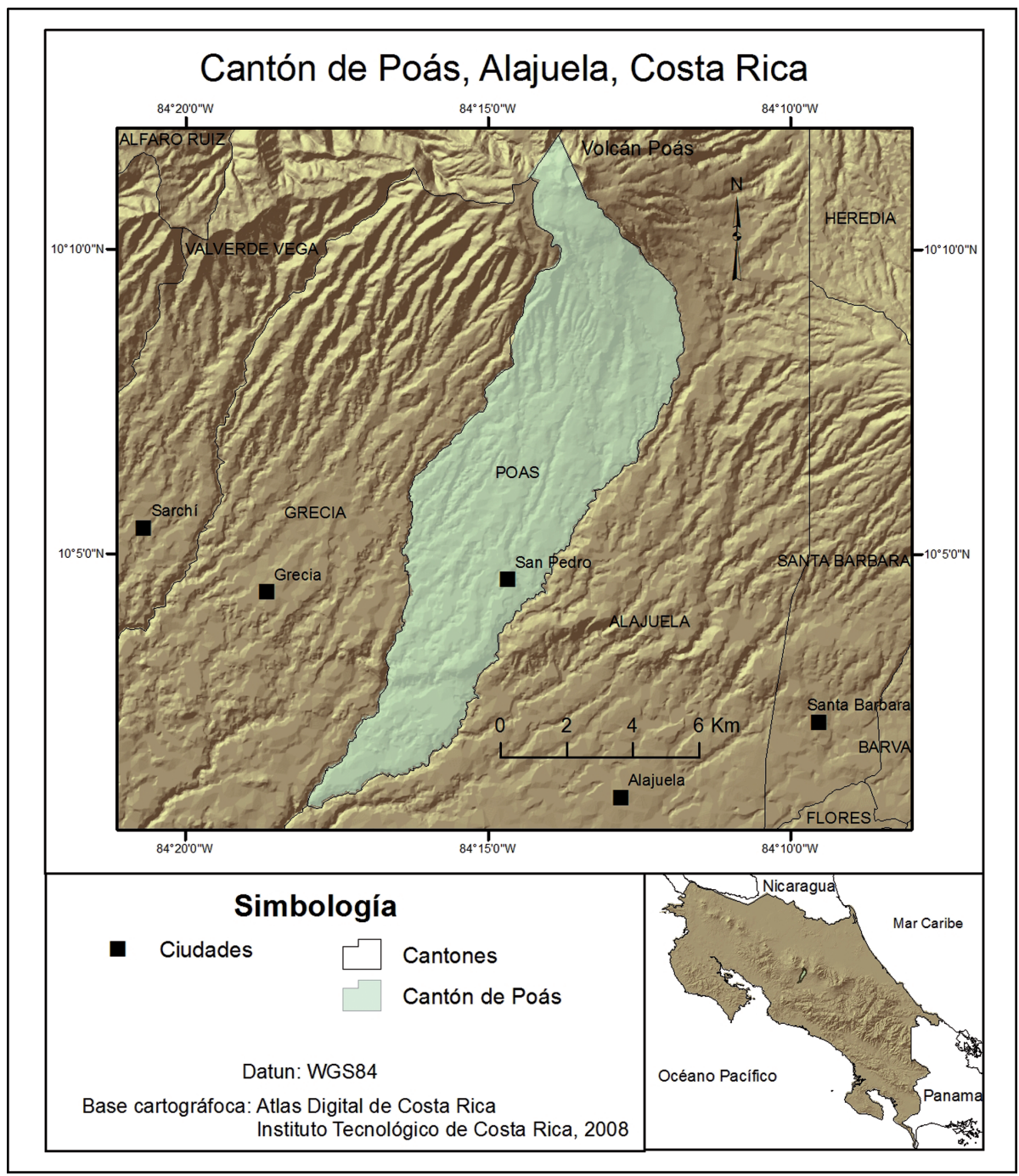

FIGURA 1. Área de estudio.

En el tema sísmico propiamente, está a disposición el Código Sísmico de Costa Rica 2010 (Poder ejecutivo, 2012), no obstante dicho código establece normas constructivas y no resulta en una referencia para ordenar el uso del suelo. Una alternativa es adaptar métodos de evaluación de la amenaza sísmica a los requerimientos del OT por medio del uso de SIG.

Por su parte, los métodos de evaluación de la amenaza sísmica pueden ser revisados en dos grupos: determinísticos y probabilísticos. A continuación se describen, de acuerdo con Benito y Jiménez (1999); McGuire (2001) y Abrahamson (2006).

Los métodos determinísticos fueron los primeros en desarrollarse y tienen por objetivo determinar la acción sísmica en un emplazamiento determinado. En líneas generales siguen los siguientes pasos:
- Determinación del área de influencia del emplazamiento e identificación de las fuentes sísmicas englobadas en ella.

- Estimación de los mayores terremotos ocurridos en el área de influencia. Identificación de los sismos característicos y definición del máximo sismo potencial a esperar en cada zona o estructura delimitada.

- Estimación de la acción sísmica en el emplazamiento causada por los máximos sismos potenciales de cada zona o del área total. Se sigue un criterio conservador ubicando dicho sismo en el punto de la zona más cercano al emplazamiento. Con leyes de atenuación se obtienen los valores de intensidades del movimiento en el emplazamiento como consecuencia de cada escenario definido.

- Determinación de la amenaza en el emplazamiento, 
tomando el máximo valor de intensidad del parámetro generado por los escenarios, con lo que la amenaza queda caracterizada por el límite superior del movimiento en el punto.
El método determinístico utiliza un valor singular de parámetro seleccionado para cada escenario. Un esquema simplificado del método se observa en la FIGURA 2.
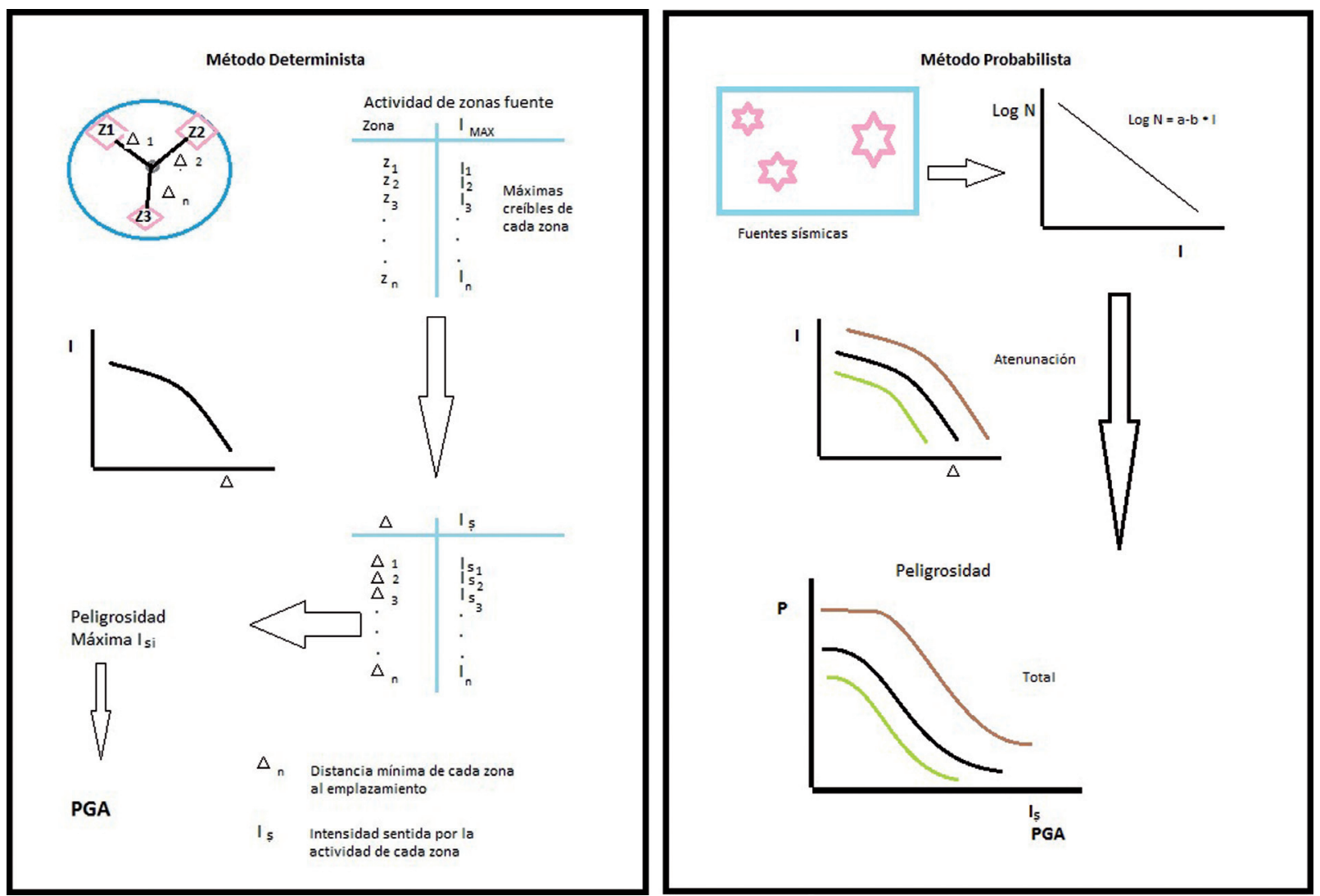

FIGURA 2. Esquema simplificado de los métodos de estimación de la amenaza sísmica. Fuente: Benito y Jiménez (1999).

El método probabilístico por su parte, considera todos los terremotos que afectan el emplazamiento y tienen en cuenta la recurrencia de los mismos. De esta forma ofrecen estimaciones de la probabilidad de excedencia para cada intensidad de movimiento esperado en el emplazamiento durante un periodo de tiempo dado, usualmente representado en curvas de probabilidad. El análisis probabilístico se puede visualizar como un gran número de análisis determinísticos. Estos métodos se pueden subdividir en paramétricos y no paramétricos, la diferencia fundamental entre unos y otros está en que en el primer caso se utilizan funciones de distribución de valores extremos como los métodos Gumbel, mientras que los no paramétricos se basan en zonas sismogénicas en las que se divide el área de influencia y el ajuste de la sismicidad de cada zona a un modelo de recurrencia, sumando posteriormente las contribuciones de todas las zonas para obtener la función de probabilidad del emplazamiento. El grupo de escenarios utilizados debe incluir únicamente sismos físicamente posibles.
Muñoz (1989) señala que el método probabilístico ofrece varias ventajas frente al determinístico, entre ellas la principal es la obtención de la distribución de probabilidades para distintos niveles de intensidad o daño frente al valor de máxima intensidad que ofrece el método determinístico. Así también resalta que con los métodos probabilísticos, la ubicación de los epicentros históricos no es tan crítica como en los métodos determinísticos y que en estos últimos, el terremoto de mayor tamaño está condicionado por la información existente, limitante que se solventa con la utilización de parámetros estadísticos de la sismicidad en cada zona, en el caso de los probabilísticos. No obstante, McGuire (2001) afirma que al enfocarse en un solo evento sísmico los métodos determinísticos aseguran que dicho evento es realista, es decir razonablemente probables, indica además que en este sentido los métodos determinísticos y probabilísticos son complementarios, ya que un análisis probabilístico puede ser confirmado con un evento determinístico que se considere para asegurar 
que una hipótesis realista ha sido considerada en el análisis.

A pesar de las ventajas de los métodos probabilísticos en este estudio se seguirá el procedimiento determinístico, tal como se sugiere en la Guía Análisis de Riesgos Naturales para el Ordenamiento Territorial(Subsecretaría de Desarrollo Regional y Administrativo, 2011), como alternativa de aproximaciones menos rigurosas como la propuestas en el IFA denominada "índice de densidad sísmica" (Decreto No 32967-MINAE). El propósito es demostrar que es posible adaptar un método robusto de evaluación sísmica a los propósitos del OT. Por otra parte, al considerar el peor escenario posible, el método determinístico ofrece la ventaja de dar sustento a decisiones sobre el uso del suelo en los Planes de Ordenamiento Territorial, evitando así el incremento del riesgo futuro por medio del aumento de la vulnerabilidad (Smith and Petley, 2007).

\section{METODOLOGÍA}

En esta investigación se parte del método determinístico para el análisis de la amenaza sísmica, por medio del cual es posible obtener valores absolutos al evaluar diferentes escenarios sísmicos basados en el conocimiento actual de las condiciones sismotectónicas de la región (González et al., 2002).

En su adaptación para el uso de Sistemas de Información Geográfica (SIG) se sigue la guía presentada para el caso de Kathmandu, Nepal (Westen et al., 2012). El procedimiento puede resumirse en los siguientes pasos:

- Identificación de posibles fuentes sísmicas próximas al cantón haciendo uso de datos sísmicos instrumentales e históricos.

- Definición de fuentes sismogénicas. Para esto se consideró la localización de epicentros, magnitudes y profundidades de los sismos instrumentales e históricos.

- Determinación del sismo máximo a esperar, siguiendo tres criterios: uno estadístico, otro empírico y el último histórico, comparando los tres criterios se toma la decisión de la magnitud a utilizar en los sismos de diseño por cada fuente.

- Cálculo de la atenuación sísmica en valores de Aceleración Horizontal Pico (PGA por sus siglas en inglés) considerando el efecto de amplificación debido a las condiciones locales del suelo.

- Conversión de los valores de PGA en valores Intensidad acorde con la escala de Intensidad Mercalli Modificada (IMM).
- Adaptación del resultado para su aplicación en el OT.

En este estudio se han definido pixeles de $5 \times 5 \mathrm{~m}$, lo que permite una resolución apropiada a escalas útiles para la planificación territorial. No obstante el detalle de los mapas geológicos determina la escala mínima de trabajo, en este caso 1:50.000.

\section{MARCO TECTÓNICO}

Costa Rica se ubica entre los límites de las placas Caribe, Cocos, Nazca y la microplaca de Panamá. Presenta un vulcanismo activos hasta el límite de la placa de Cocos con la microplaca de Panamá, y una alta sismicidad de profundidad superficial e intermedia (Climent et al., 2008). Según Montero (2001), el territorio costarricense se ubica sobre dos provincias sismotectónicas, la mitad norte del país se encuentra dentro de la placa Caribe y la parte sur sobre la microplaca de Panamá (FIGURA 3).

Por su parte, la placa de Cocos subduce bajo la Caribe a una velocidad de 7,8 cm/año frente a la costa Pacífica de Costa Rica (Protti et al., 2012) pero con dos morfologías diferentes. Al norte del país, la forma es suave y de edades entre 22-24 Ma, mientras que la subducción bajo la parte central y sureste del país se presenta rugosa y con edades entre 15-19 Ma (Climent et al., 2008). Esto se relaciona con el hecho de que la litosfera de la placa de Cocos creada en la Dorsal del Pacífico subduce por debajo de la parte norte del país, mientras que la parte de la placa de Cocos creada en la Dorsal de Galápagos subduce por debajo de la parte sur y central (Protti et al., 2014). Al sur de la punta Burica se encuentra la zona de fractura de Panamá que constituye el límite entre las placas de Cocos y Nazca (FIGURA 3).

De acuerdo con Montero (2001) el área de estudio del presente trabajo se ubica dentro del denominado Cinturón Deformado del Centro de Costa Rica, área que marca el límite norte de la microplaca de Panamá, se trata de una zona de deformación de un ancho de más de $100 \mathrm{~km}$ sobre el centro del país y que continúa en el Cinturón Deformado del Norte de Panamá. Según este autor, al oeste de la Cordillera Volcánica Central predominan fallas normales y de rumbo, orientadas en patrones principalmente NW-SE, y N-S.

Dado que en el registro histórico no se reportan eventos de origen interplaca que hayan afectado de manera considerable el área de estudio (posiblemente debido la distancia a la zona de subducción) y por el contrario se han presentado varios terremotos de tipo intraplaca, 
en esta investigación sólo se consideran las fuentes sísmicas asociadas con el fallamiento local, lo que es congruente con otros estudios de amenaza realizados en el Valle Central de Costa Rica (Alvarado et al., 1988; Montero y Morales, 1988; Climent et al., 2006).

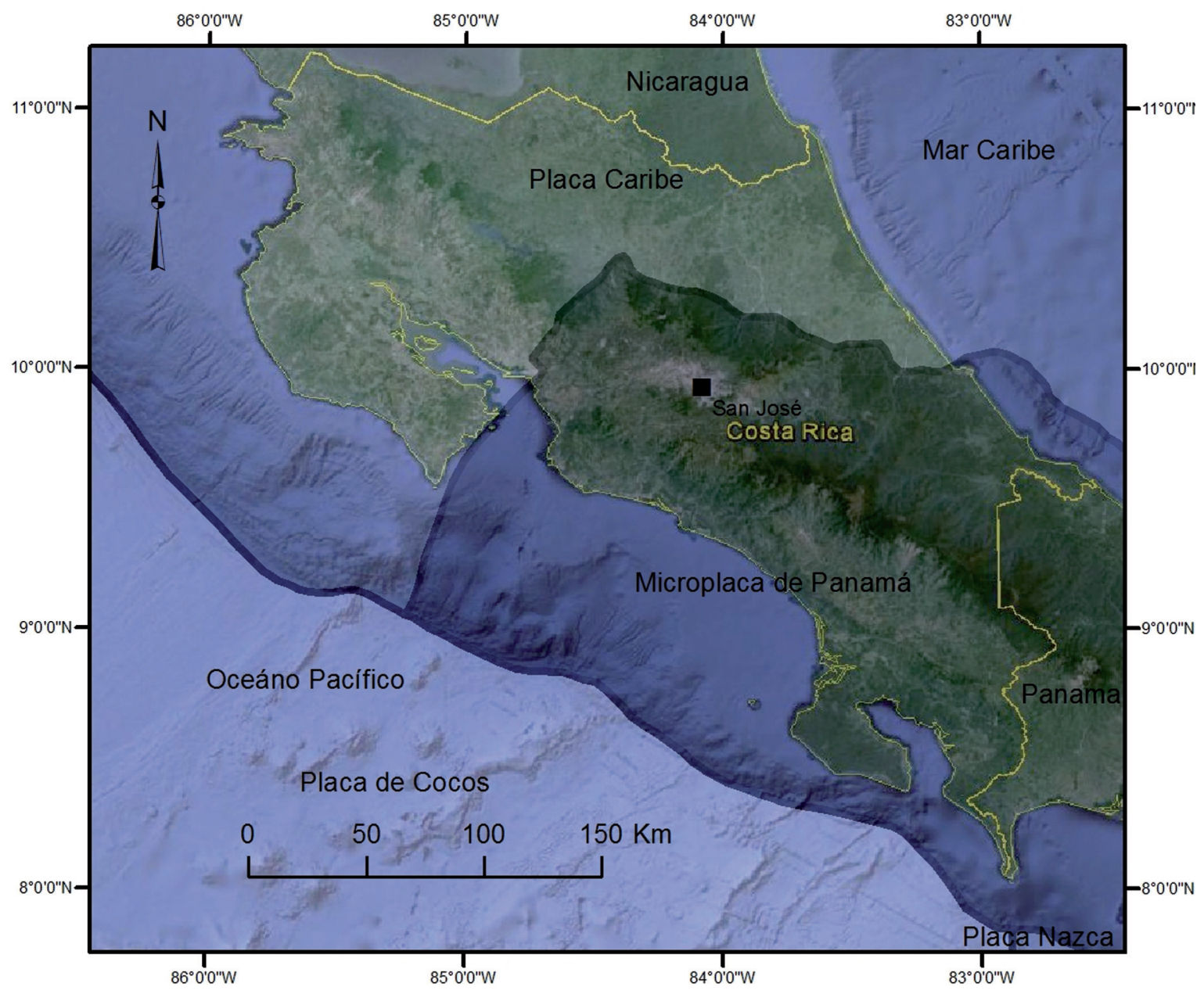

FIGURA 3. Mapa tectónico simplificado de Costa Rica. Fuente: Climent et al. (2008).

De acuerdo con Climent et al. (2006) y Montero et al. (2010), las fallas que se consideran activas próximas al área de estudio son (FIGURA 4):

Falla Carbonera: falla de rumbo NNW dextral, de unos $6,5 \mathrm{~km}$ de longitud. Su continuidad hacia el sur no es clara debido a los depósitos volcánicos del Cuaternario.

Falla Viejo-Aguas Zarcas: falla en tijera de rumbo NNW-SSE, que inicia cerca del volcán Viejo, de una longitud $25 \mathrm{~km}$.

Falla Ángel: falla segmentada de rumbo que varía en su rumbo entre $\mathrm{N} 70^{\circ} \mathrm{W}$ y N-S, la misma se extiende por más de $20 \mathrm{~km}$ según el trazo propuesto por Montero et al. (2010). La falla muestra escarpes facetados al este y al oeste, así como valles lineales localmente, ríos adaptados o desplazados y sillas de falla, y parece tener componente dextral y normal (Barquero, 2009).

Falla Sabanilla: fue definida por primera vez por Montero et al. (2010), la consideran responsable de los terremotos de 1911 y 1912 . De acuerdo con estos autores, la falla se extiende alrededor de $25 \mathrm{~km}$ con un rumbo NW, al este la falla tiene un trazo incierto y pierde continuidad al acercarse a la falla Alajuela, pero alcanza a atravesar el cantón de Poás.

Falla de Alajuela: el escarpe de esta falla ha sido relacionado con un pliegue asociado a la propagación inversa de la falla, que tiene un rumbo ESE y WNW, extendiéndose por unos $20 \mathrm{~km}$. 


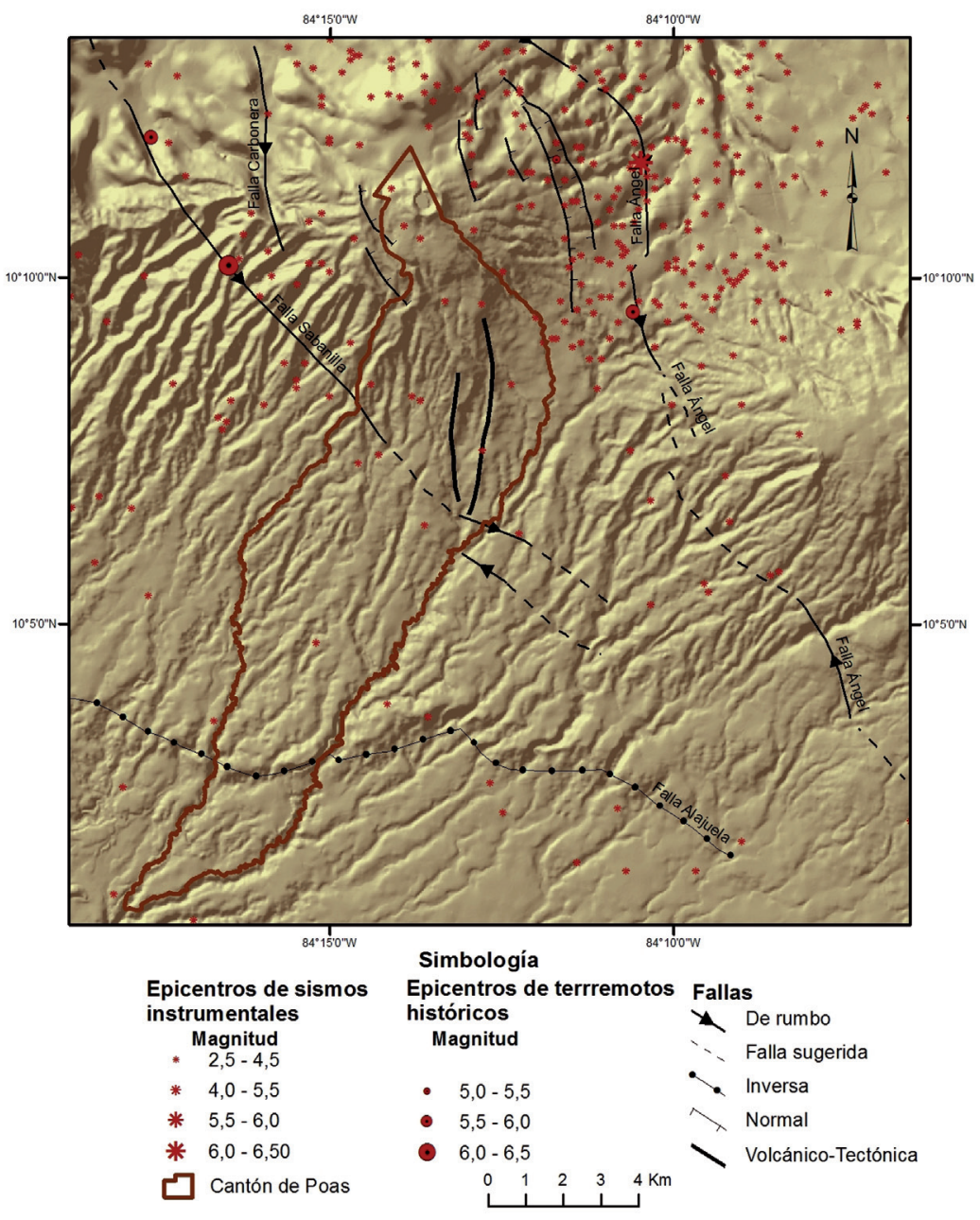

FIGURA 4. Fallas y actividad sísmica presentes en los alrededores del cantón de Poás. La localización regional puede apreciarse en la FIGURA 1. (Fuente: sismicidad instrumental de OVSICORI-UNA y fallas de Montero et al., 2010).

La morfología del cantón y zonas aledañas es caracterizada por su ubicación en la ladera suroeste del Volcán Poás, donde sobresalen la cima del edificio volcánico, los conos piroclásticos de Sabana Redonda y el anticlinal de Alajuela.

\section{ANÁLISIS DE SISMICIDAD}

\section{Sismicidad Instrumental}

Para el análisis de la sismicidad se contó con los registros del OVSICORI, desde 1984 hasta el 2013 (OVSICORI, 2013). Se utilizó como referencia la zona sísmica "Cordillera Volcánica Central y Valle Central" o Zona C6 (Climent et al., 2008). Los sismos analizados son aquellos con profundidad $\leq 30 \mathrm{~km}$ y magnitud $\mathrm{ML} \geq 2.5$, valor considerado como la magnitud mínima representativa de acuerdo con la relación Gutenberg-
Richter elaborada para la zona de influencia sísmica. Estos datos fueron complementados con el catálogo del Centro Sismológico Internacional (International Seismological Centre, 2014). La FIGURA 5 muestra la distribución espacial de la sismicidad y resalta los sismos históricos sobresalientes para la zona.

Como se puede apreciar en la FIGURA 5, los sismos tienden a agruparse en relación con las estructuras neotectónicas. En este sentido se observa una concentración de epicentros próximos al límite noreste del cantón, relacionados con la Falla Ángel. El otro agrupamiento cercano (al sur) se relaciona con el enjambre sísmico de Puriscal de 1990 originado en dos sistemas de fallas: San Antonio-Picagres de movimiento predominantemente dextral y la falla Víbora que presenta un movimiento oblicuo dextralinverso (Montero et al., 1990). 


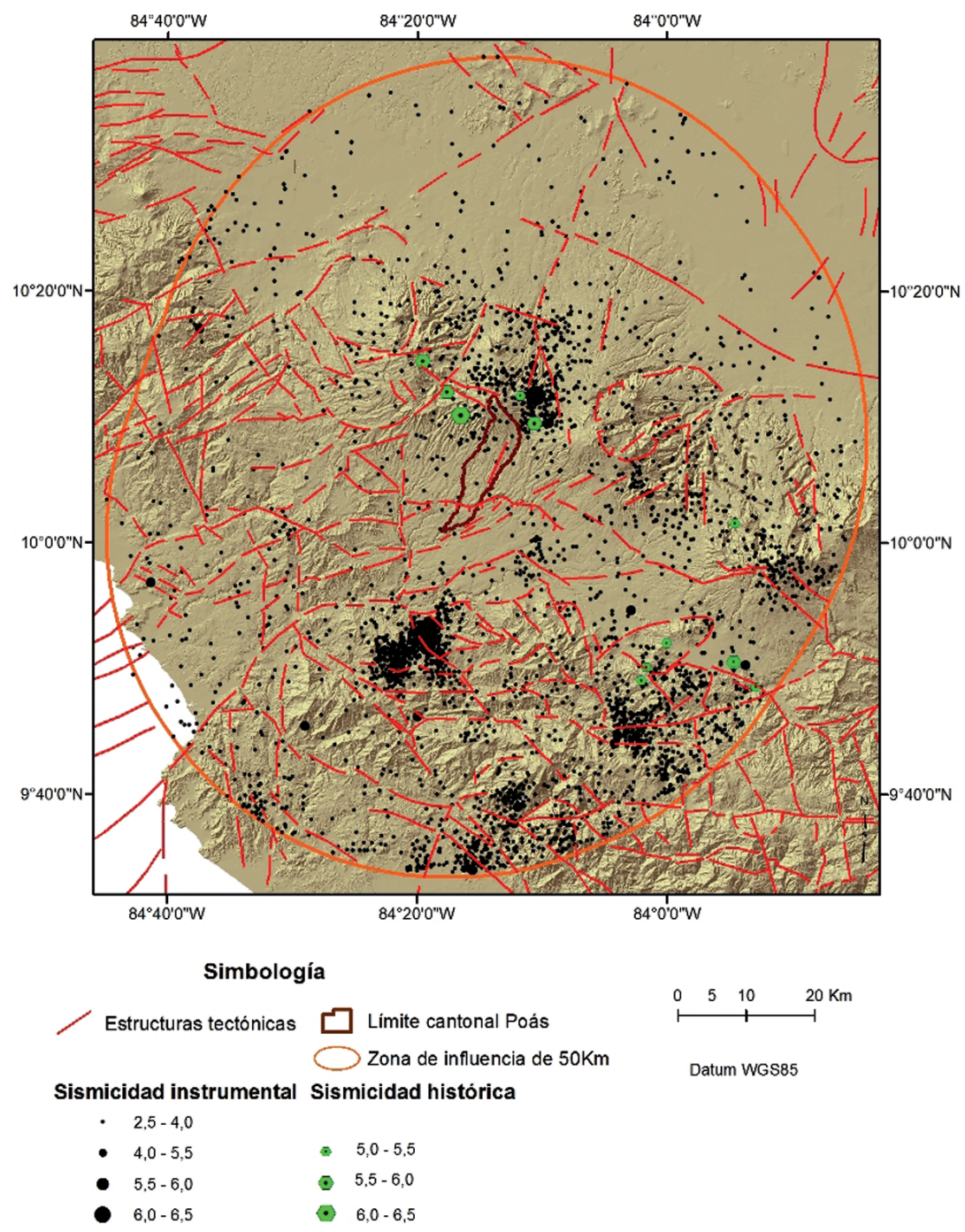

FIGURA 5. Tectónica y sismicidad relacionada con el cantón. Fuente OVSICORI (2013).

La agrupación ubicada al sureste está relacionada con la falla Aguacaliente cuya traza principal es de rumbo WNW y se localiza sobre el borde sur de los valles de Coris y de El Guarco y se le considera responsable de los enjambres sísmicos ocurridos en años recientes (Montero y Kruse, 2006). Finalmente, al extremo sur del área de influencia sísmica se encuentra un agrupamiento sísmico posiblemente relacionado con la falla Candelaria, estructura de rumbo dextral que posee una longitud aproximada de $75 \mathrm{~km}$.

\section{Sismicidad Histórica}

La información histórica utilizada responde a las recopilaciones hechas en trabajos previos (Climent et al., 2006; Montero et al., 2010; Montero, 1989; Montero et al., 1997; Montero y Morales, 1988; Ramírez et al., 1996; Rojas et al., 1993). La distribución de los epicentros históricos en el Valle Central de Costa Rica, se relaciona principalmente con la falla Aguacaliente y Coris en el sector este del Valle Central y con las fallas Viejo-Aguas Zarcas, Ángel y Sabanilla en torno al volcán Poás (FIGURA 5).

Terremotos que han afectado al cantón de Poás: el cantón de Poás ha sido afectado por varios terremotos entre los que destacan el del 15 de febrero de 1772, el de Fraijanes del 30 de diciembre de 1888, el del 28 de agosto de 1911, el del 6 de junio de 1912 y el del 1 de setiembre de 1955 , los cuales se detallan en el TABLA 1. Adicionalmente, Morales et al. (1992) reportan un terremoto ocurrido el 28 de marzo de 1851 de características similares al terremoto de Fraijanes de 1888 pero que causó menor destrucción, asociado con la misma fuente sísmica de este último. 
TABLA 1. Principales terremotos que han afectado al cantón de Poás

\begin{tabular}{|c|c|c|c|c|}
\hline \multirow{2}{*}{ Fecha } & Coordenadas & \multirow{2}{*}{ Magnitud } & \multirow{2}{*}{ Intensidad máxima } & \multirow{2}{*}{ Posible falla de origen } \\
\hline & Lat. N Long. W & & & \\
\hline $15 / 02 / 1772$ & --------- ----------- & $\mathrm{M} \sim 5,5-6,0$ & VII & Ángel o Sabanilla \\
\hline $30 / 12 / 1888$ & $10^{\circ} 09.5^{\prime} 84^{\circ} 11.0^{\prime}$ & ML 5,7 & VIII & Ángel \\
\hline 28/08/1911 & $10^{\circ} 12.0^{\prime} 84^{\circ} 18.0^{\prime}$ & Ms 6,8 & VII & Sabanilla \\
\hline 06/06/1912 & $10^{\circ} 10.0^{\prime} 84^{\circ} 17.0^{\prime}$ & Ms 5,1 & VII & Sabanilla \\
\hline 01/09/1955 & $10^{\circ} 14.5^{\prime} 84^{\circ} 19.5^{\prime}$ & Ms 5,5 & VII & Viejo-Aguas Zarcas \\
\hline 08/01/2009 & $10^{\circ} 11.5^{\prime} 84^{\circ} 11.0^{\prime}$ & Mw 6,1 & IX & Ángel \\
\hline
\end{tabular}

Fuente: Modificado de Montero et al. (2010)

El terremoto que ha causado más afectación en el cantón de Poás, de acuerdo con las zonas mesosísmicas presentadas por Montero et al. (2010) para los terremotos mencionados, es el terremoto de Fraijanes de 1888. Para este terremoto Alvarado et al. (1988) reportan una intensidad máxima de VIII que cubre una amplia sección al norte del cantón (alrededor de los conos monogenéticos de Sabana Redonda). En aquella época Henri Pittier registró la ocurrencia de deslizamientos de varios tamaños, pequeños a lo largo de las vías de acceso a la zona y grandes como el que daría lugar a la Laguna de Fraijanes, así como un flujo de derrubio de más de 500 $\mathrm{m}$ que arrasó varias casas a su paso y además se reportó la ocurrencia de cinco muertos (González, 1910).

Morales et al. (1992) consideraron que la fuente más probable fue la falla de Alajuela y proponen un epicentro ubicado en una depresión que muestra alineamientos con aparente control estructural, aspecto que podría sugerir un origen diferente al de la falla citada. Con base en estos indicios y en el nuevo trazo de la falla Ángel, Montero et al. (2010) afirman que ésta última es la fuente del terremoto de Fraijanes (TABLA 1). Un aporte valioso al estudio de este evento es el mapa de isosistas (FIGURA 6), inferido a partir de los informes de Pittier (Morales et al., 1992).

Además de estos eventos históricos, el cantón Poás ha sido afectado por un evento contemporáneo ocurrido el 08 de enero de 2009 , que ha sido denominado "Terremoto de Cinchona". Dicho sismo tuvo una magnitud Mw 6,1, a una profundidad de $6 \mathrm{~km}$, con una intensidad máxima IX, y fue originado por la falla Ángel, como resultado de un fallamiento oblicuo en dirección aproximadamente NW - SE, cuya ruptura se extendió por unos $12 \mathrm{~km}$ en la horizontal (Barquero, 2009).

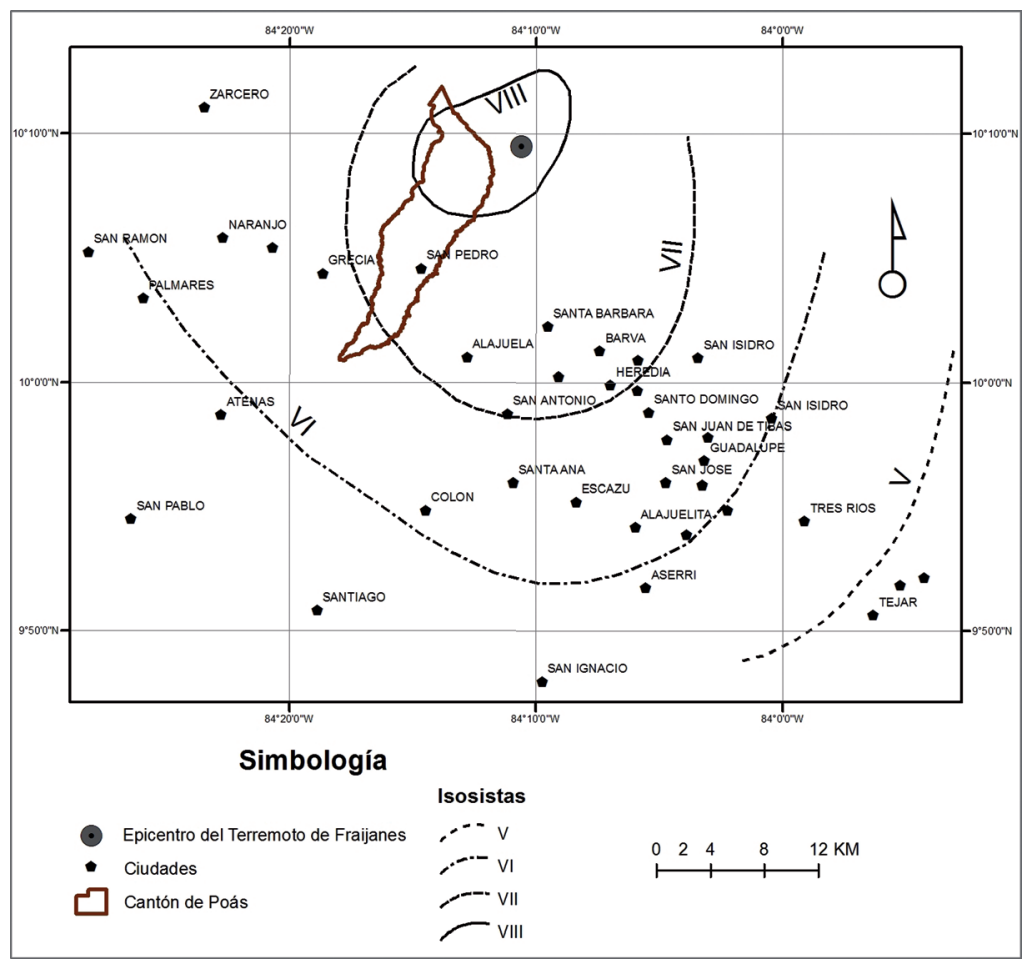

FIGURA 6. Mapa de isosistas del terremoto de Fraijanes de 1888 (Modificado de Morales et al., 1992). 
Como resultado de la sacudida sísmica del evento del 08 de enero de 2009 ocurrieron alrededor de 4.600 deslizamientos de distintas dimensiones y tipos (Barrantes et al., 2013), que aumentaron significativamente los daños. Este sismo cobró la vida de 25 personas y afectó infraestructuras como puentes, caminos, viviendas, iglesias, líneas de transmisión eléctrica y las represas hidroeléctricas de Toro II y Cariblanco, con pérdidas totales estimadas en 280.765 millones de colones (Laurent, 2009) alrededor de US\$510.500. La FIGURA 7 presenta las isosistas establecidas por la Red Sismológica Nacional (RSNUCR-ICE), donde es posible apreciar intensidades MM de VI, VII y VIII en el cantón de Poás.

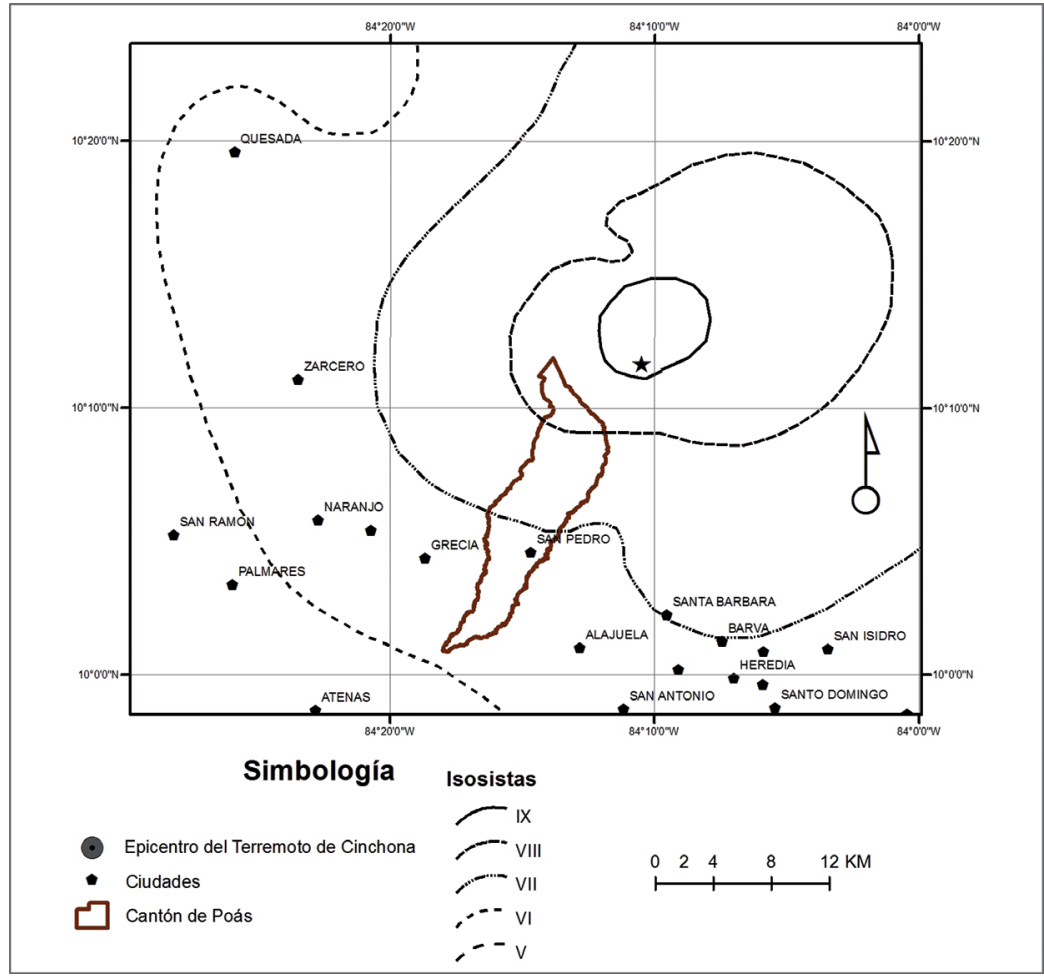

FIGURA 7. Mapa de isosistas del terremoto de Cinchona de 2009 (Modificado de Barquero, 2009).

En el caso de los terremotos de 1911 y 1912 a los que Montero et al. (2010) relaciona con la falla Sabanilla, es posible que debido a la profundidad estimada (TABLA 1) éstos solo alcanzaron una intensidad máxima de VII en el límite noroeste del cantón de Poás.

Como resultado de esta revisión se concluye que las principales fuentes de amenaza sísmica en el cantón Poás son las fallas Ángel y la falla Sabanilla (FIGURA 4), tanto por su proximidad al cantón como por su potencial sísmico.

\section{VALORACIÓN DE LA AMENAZA SÍSMICA}

De acuerdo con la distribución de los epicentros de los sismos históricos y sus análisis posteriores, particularmente la distribución de las isosistas de máxima intensidad presentada por Montero et al. (2010), el área de estudio ha sido afectada por siete terremotos, de los cuales los relacionados con las fallas Sabanilla y Ángel han sido los más destructivos en el Cantón (TABLA 1).

Las nuevas trazas de las fallas propuesta por Montero et al. (2010) implica una aumento de la amenaza sobre el cantón de Poás ya que la falla Sabanilla lo atravesaría, y la Falla Ángel se acerca a su límite noreste (FIGURA 4). Para el caso de ambas fallas la distribución de epicentros instrumentales tiende a alinearse validando sus trazos propuestos.

\section{Sismos de diseño}

Haciendo uso del trazo de la falla Sabanilla se ha estimado su extensión en 22,7 km, desde Bajos del Toro hasta poco antes de Carrizal de Alajuela, considerando que el trazo está mejor diferenciado en el sector norte y central, donde tiene un aparente movimiento dextral (Montero et al., 2010). Estos autores le han atribuido a 
esta falla los terremotos de 1911 y 1912, ubicando sus epicentros a una distancia aproximada de 5,2 km entre ellos.

A pesar de atribuir el sismo de 1955 a la falla ViejoAguas Zarcas (TABLA 1), su epicentro también se distancia aproximadamente a $5,2 \mathrm{~km}$ del sismo de 1911. En este sentido vale aclarar que otros autores le atribuyen estos tres sismos a la falla Viejo - Aguas Zarcas (Montero y Morales, 1988). Asumiendo que los sismos de 1911, 1912 y 1955 siguen un patrón temporal y espacial es posible suponer que la falla ya ha roto en su extremo noroeste y estaría próxima a hacerlo en su segmento sureste (FIGURAS 4 y 5).

Con base en este razonamiento se decide ubicar el epicentro del sismo de diseño a 5,2 km al sureste del epicentro del terremoto de 1912 en un sector bien establecido del nuevo trazo de la falla Sabanilla, lo cual es consecuente con el método determinista, pues el epicentro se ubicaría dentro del cantón de Poás, lo que constituiría el peor escenario posible. La profundidad asignada al sismo de diseño se establece a $8 \mathrm{~km}$, valor que representa la profundidad media de los sismos ubicados en un corredor a $5 \mathrm{~km}$ de ancho a lo largo de esta falla.

Para determinar la magnitud máxima de este sismo de diseño se utilizan las relaciones empíricas entre longitud de la falla y magnitud de Wells and Coppersmith (1994), quienes para fallamiento de rumbo proponen la siguiente ecuación:

$$
M=5,1+1,12 \log (S R L)
$$

Donde: $M$ es la magnitud momento, y $S R L$ es la Longitud de Ruptura Superficial.

De esta manera se obtiene una magnitud potencial máxima de Mw 6,7. Como se aprecia en la TABLA 1, el mayor evento histórico para esta falla es estimado en Ms 6,8 (terremoto de 1911). Para homogenizar la escala se aplicaron las formulas aportadas por Rojas et al. (1993), con base en las cuales se obtiene una magnitud $\mathrm{Mw}$ 6,7.

Por su parte, la falla Ángel se extiende por 22,6 km, con movimiento predominantemente dextral. Su traza, que inicia a 1,6 km del volcán Congo y finaliza al norte de Birrí, está dividida en tres segmentos, noroeste, central y sureste (Montero et al., 2010). Al tramo noreste se le atribuye el terremoto de 2009 y al tramo central el de 1888.
Considerando que el tramo sureste de la falla es incierto y tiende a alejarse del cantón (FIGURA 4) y que el tramo central es el más próximo al cantón y tuvo su último sismo fuerte en 1888, siguiendo los postulados del ciclo sísmico (Ruegg, 1994), es razonable pensar que ha transcurrido el tiempo necesario para que en este segmento haya acumulado la energía suficiente para generar un evento sísmico similar, por lo que se decide ubicar el sismo de diseño en el mimo lugar del epicentro del terremoto de 1888. De igual manera, para determinar su profundidad se siguió el criterio de la media de profundidades en una zona de influencia de $5 \mathrm{~km}$ a lo largo de la falla, quedando el hipocentro a $6,7 \mathrm{~km}$

Al aplicar la ecuación [1] se obtuvo una magnitud potencial máxima para esta falla de $\mathrm{M}_{\mathrm{w}}$ 6,7. Cabe señalar que el terremoto de 1888 tuvo una magnitud de $M_{L}$ 5,7 que, de acuerdo con las formulas aportadas por Rojas et al. (1993), equivale a $M_{w} 6,2$, valor que resulta menor a la magnitud potencial evaluada por la longitud de la falla.

El resumen de los parámetros considerados para la determinación de los sismos de diseño en cada una de las fuentes sismogénicas se presenta en la TABLA 2.

TABLA 2. Resumen de parámetros para posibles sismos de diseño

\begin{tabular}{|c|c|c|c|}
\hline Falla & Epicentro & $\begin{array}{c}\text { Profundidad } \\
(\mathrm{Km})\end{array}$ & $\begin{array}{c}\text { Magnitud } \\
\left(M_{w}\right) \\
\end{array}$ \\
\hline Sabanill & $84^{\circ} 14^{\prime} 12^{\prime \prime} \mathrm{W} \quad 10^{\circ} 7^{\prime} 37^{\prime \prime} \mathrm{N}$ & $\begin{array}{c}8^{1} \\
18^{3}\end{array}$ & $\begin{array}{l}6,7^{2} \\
6,7^{3}\end{array}$ \\
\hline Ángel & $84^{\circ} 10^{\prime} 46^{\prime \prime} \mathrm{W} \quad 10^{\circ} 9^{\prime} 43^{\prime \prime} \mathrm{N}$ & $\begin{array}{l}6,7^{1} \\
15^{3}\end{array}$ & $\begin{array}{l}6,7^{2} \\
6,2^{3}\end{array}$ \\
\hline Mmax & Relación Gutenberg & -Richter & 6,8 \\
\hline
\end{tabular}

${ }^{1}$ Profundidad media de los sismos ubicados dentro de una zona de influencia de la falla de $5 \mathrm{~km} ;{ }^{2}$ Obtenido por medio de una relación empírica entre la longitud de ruptura superficial de la falla y la magnitud; ${ }^{3}$ Sismos histórico mayor asociado con la falla.

Para complementar el criterio histórico y de longitud de falla en la determinación de la magnitud del sismo de diseño, se hace uso de una aproximación estadística basada en la relación Gutenberg-Richter:

$$
\log N=a-b M
$$

Donde: $M$ es la magnitud, $a$ y $b$ son constantes, a partir de las cuales es posible obtener el sismo máximo posible asumiendo que $\mathrm{M}_{\mathrm{Max}}=\mathrm{a} / \mathrm{b}$. 
De acuerdo con el registro instrumental utilizado, así como de la línea de mejor ajuste para la relación frecuencia - magnitud (FIGURA 8), la magnitud máxima a esperar es de $\mathrm{M}_{\max }=6,2 \mathrm{Ml}$, que corresponde a $\mathrm{M}_{\mathrm{w}} 6,8$ de acuerdo con las correlaciones de Rojas et al. (1993) (TABLA 2).

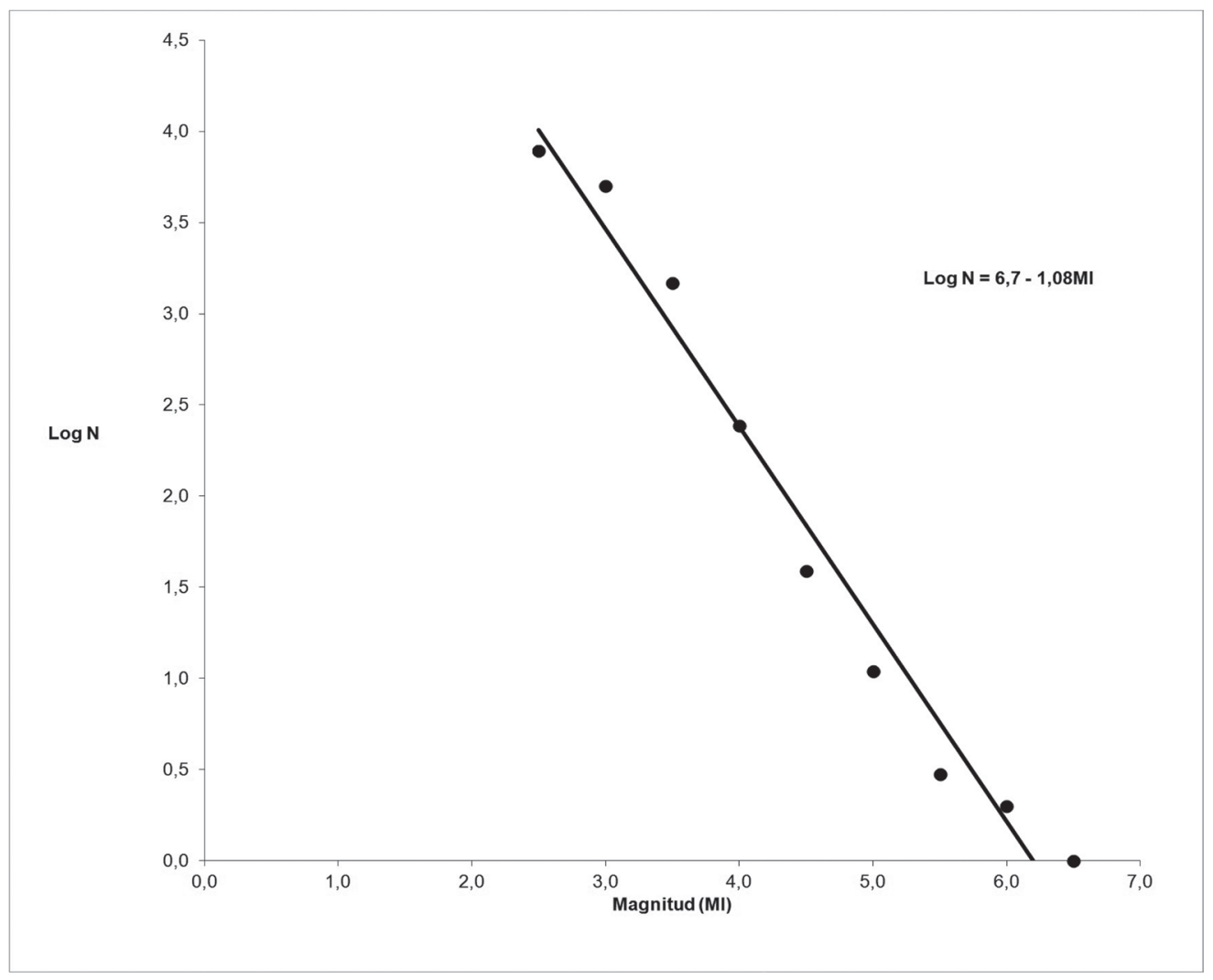

FIGURA 8. Línea de mejor ajuste para la relación frecuencia-magnitud.

En resumen, los sismos de diseño seleccionados corresponden a los siguientes: uno generado por la falla Sabanilla de magnitud $M_{w} 6,7$ y profundidad de $8 \mathrm{~km}$ y otro por la falla Ángel de $\mathrm{M}_{\mathrm{w}} 6,7$ a una profundidad de $6,7 \mathrm{~km}$.

\section{Determinación de intensidades máximas}

Existen diferentes modelos empíricos de atenuación sísmica; en la búsqueda de los más apropiados para Costa Rica, Climent et al. (2008) comparan ocho y concluyen que para fuentes superficiales intraplaca, como es el caso del área de estudios, los modelos propuestos por Zhao et al. (2006), Schmidt et al. (1997) y Climent et al. (1994) resultan los más confiables. En esta investigación se utilizará la ley de atenuación para América Central desarrollada por Climent et al. (1994), en la determinación de los valores de PGA para un emplazamiento. Su ecuación se muestra a continuación:

$$
\begin{gathered}
\ln A=-1,687+0,553 * M-0,537 * \ln (r) \\
-0,00302 * r+0,327 * S+\ln \in
\end{gathered}
$$

Donde:

A: Aceleración horizontal pico en $\mathrm{m} / \mathrm{s}^{2}$ (PGA)

$M$ : Magnitud momento (Mw)

$r$ : Distancia hipocentral

$S$ : $\quad$ Equivale a 0 para sitios de roca y 1 para suelos

ln€: Término relacionado con el error de estimación equivalente a 0,75

Para incluir el efecto local en esta fórmula se siguen las recomendaciones del método RADIUS - Risk Assessment Tools for Diagnosis of Urban areas against Seismic Disasters - (Subsecretaría de Desarrollo Regional y Administrativo, 2011). Para su implementación las amplificaciones sísmicas fueron inferidas a partir del mapa geológico detallado, por medio de una adecuación de los valores presentados en la TABLA 3 .

El primer paso realizado fue la fusión de dos mapas geológicos disponibles, el primero el mapa presentado por Ruiz et al. (2010) que responde a un trabajo depurado y actualizado pero a una escala más general 
y el otro elaborado por la Escuela Centroamericana de Geología (2005) del cual se obtuvo información más detallada para formaciones de limitada extensión. Una vez integrados y validados topológicamente, las unidades geológicas fueron clasificadas en factores de atenuación, acorde con la TABLA 3.

TABLA 3. Factores de amplificación sísmica para distintos tipos de materiales

\begin{tabular}{ccc}
\hline Código & Descripción & Factor de amplificación \\
\hline 0 & Sin información & 1,00 \\
1 & Roca dura & 0,55 \\
2 & Roca blanda & 0,70 \\
3 & Suelo medio & 1,00 \\
4 & Suelo blando & 1,30 \\
\hline
\end{tabular}

Fuente: (Westen, et al., 2012).
A partir del mapa de amplificación y de las distancias epicentrales estimadas para cada uno de los sismos de diseño, se procedió a calcular los valores de PGA para cada pixel, por medio de la ley de atenuación para América Central. Los valores obtenidos para el caso del sismo de diseñeo en la falla Ángel estuvieron en el rango de $300 \mathrm{~cm} / \mathrm{s}^{2}$ a $750 \mathrm{~cm} / \mathrm{s}^{2}$ y de $350 \mathrm{~cm} / \mathrm{s}^{2}$ a $760 \mathrm{~cm} / \mathrm{s}^{2}$ para el sismo de diseño sobre la falla Sabanilla. Como se aprecia en la FIGURA 9 en la mayor parte del cantón se esperarían valores de PGA entre $400 \mathrm{~cm} / \mathrm{s}^{2}$ y $600 \mathrm{~cm} / \mathrm{s}^{2}$. Las áreas donde se presentarían valores mayores a 600 $\mathrm{cm} / \mathrm{s}^{2}$ corresponden con efectos locales de amplificación sísmica resultado del factor de amplificación sísmica asignado a antiguos lahares y a los depósitos de tefra de la unidad Cima Volcán Poás (zonas café oscuro en la FIGURA 9).
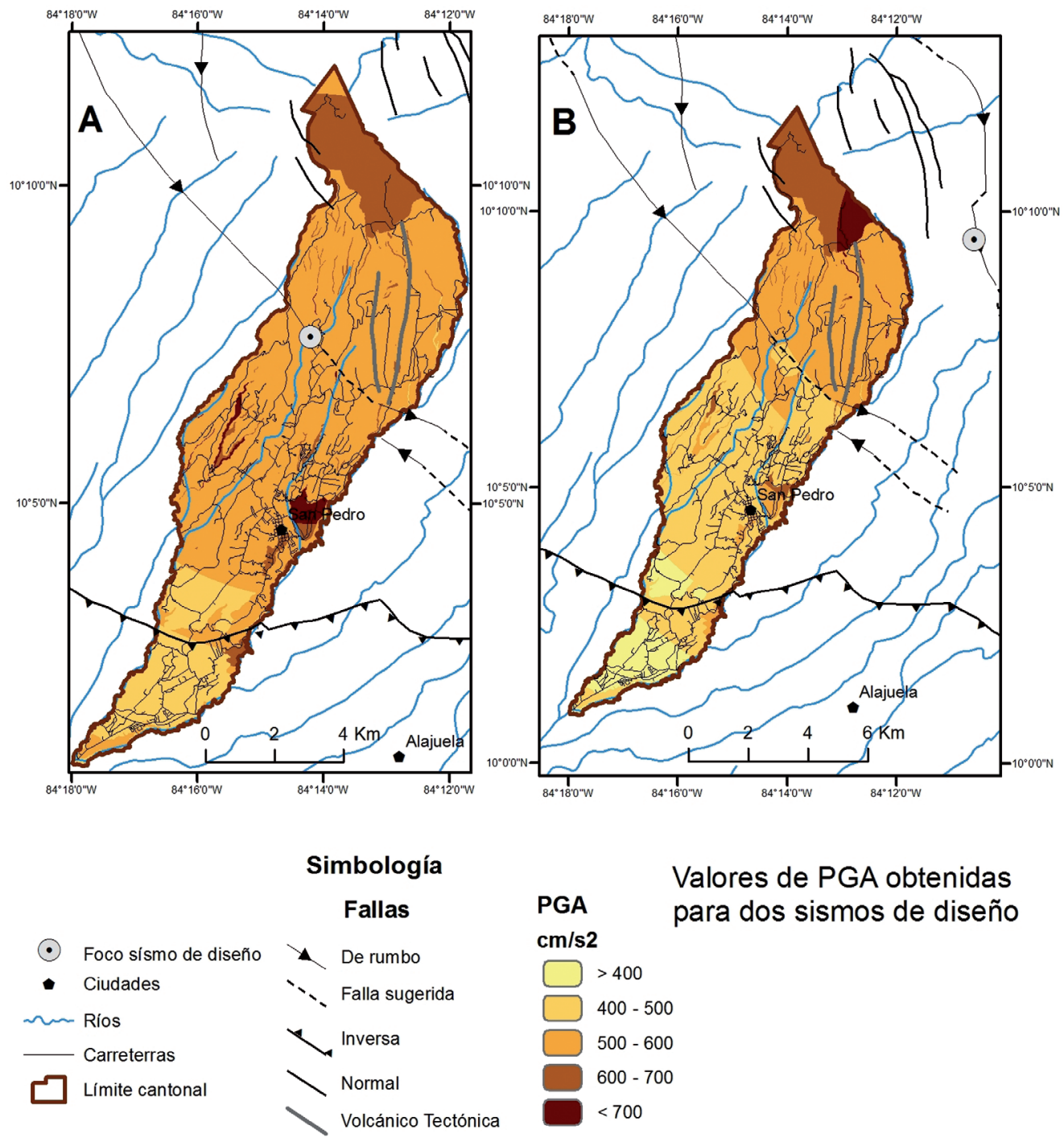

Valores de PGA obtenidas para dos sismos de diseño

$\mathrm{cm} / \mathrm{s} 2$

localizado en la falla Ángel sobre el antiguo epicentro del terremoto de 1888. 
Para una fácil comprensión e implementación de los resultados por parte de los planificadores y tomadores de decisiones, se propone transformar los valores de PGA a Intensidad Mercalli Modificada (IMM), para lo cual es posible utilizar la ecuación empírica de Trifunac and Brady (1975):

$$
I M M=3,33 \log (P G A)-0,47
$$$$
\text { Para intensidades } \mathrm{IV}<\mathrm{MMI}<\mathrm{X}
$$

No obstante, para el caso de Costa Rica, se encuentran disponibles las ecuaciones desarrolladas por Linkimer (2008) para varios rangos de intensidades y su contexto tectónico- Las utilizadas en el presente estudio corresponden a las siguientes:

$$
\begin{gathered}
I M M=2.69 \log (P G A)+0.56 \\
\text { Para intensidades } \mathrm{II}<\mathrm{MMI}<\mathrm{VII} \\
I M M=3.82 \log (P G A)-1.78
\end{gathered}
$$

Para intensidades $\mathrm{V}<\mathrm{MMI}<\mathrm{VII}$

$$
I M M=2.50 \log (P G A)+0.76
$$

Para sismos superficiales e intensidades $\mathrm{II}<\mathrm{MMI}<\mathrm{VII}$

Donde: IMM es la Intensidad Mercalli Modificada y PGA es la Aceleración Horizontal Pico.

Al aplicar las formulas se comprobó lo indicado por Linkimer (2008) quién mostró que la fórmula de Trifunac and Brady (1975) produce valores mayores a las formulas propuestas para el país. Por ejemplo, en este estudio se encontró que alrededor de un $90 \%$ del área de estudio presentaría una intensidad IX con la fórmula de Trifunac and Brady (1975) [4], lo que aparenta ser una sobrestimación para un sismo de magnitud $\mathrm{M}_{\mathrm{w}} 6,7$. De las fórmulas propuestas por Linkimer $(2008)[5,6,7]$, la que presentó una distribución acorde con las isosistas del terremoto de 1888 (FIGURA 6) y la máxima isosista alcanzada por el terremoto de Cichona (FIGURA 7) fue la ecuación desarrollada para intensidades de entre $\mathrm{V}$ y VII [6].

Los mapas de intensidades máximas esperadas para los sismos de diseño en el cantón se observan en la FIGURA 10. Como sería de esperar, el sismo potencialmente más peligroso para el área de estudio corresponde con uno originado en la falla Sabanilla con epicentro ubicado dentro del cantón de Poás y profundidad superficial.

A su vez, los mapas de intensidades (FIGURA 10) pueden ser trasformados a una escala cualitativa acorde con las clases utilizadas en el cálculo del IFA (factor Amenazas Naturales), evitando de esta manera el uso del "índice de densidad sísmica" que está establecido en el Decreto $\mathrm{N}^{\circ}$ 32967-MINAE. En la FIGURA 11 se presenta la transformación del mapa B de la FIGURA 10 a manera de ejemplo, donde las clases 3 y 4 del IFA se representan como una amenaza sísmica moderada y alta. De esta forma se demuestra cómo es posible adaptar un método sísmico robusto para su uso en la planificación territorial.

\section{DISCUSIÓN DE RESULTADO}

La selección de los datos sísmicos utilizados en esta investigación (profundidades $\leq 30 \mathrm{~km}$ y magnitudes $\mathrm{M}_{\mathrm{L}} \geq 2,5$ ) resulta similar a los $<34 \mathrm{~km}$ de profundidad $\mathrm{y}_{\mathrm{w}}>3.5$ de magnitud utilizados para la estimación de la amenaza sísmica en Costa Rica, en el marco del proyecto Resis II (Climent et al., 2008).

Por otra parte, las estimaciones de magnitud realizadas en esta investigación para los sismos de diseño (magnitud $M_{w} 6,7$ ) en ambas fuentes sismogénicas, es congruente con la estimación de la amenaza sísmica para el Proyecto Hidroeléctrico Toro 3 del Instituto Costarricense de Electricidad (Climent et al., 2006) que se localiza en cercanías del área del presente estudio. Así mismo, en el informe del proyecto Evaluación de la Amenaza Sísmica en Centroamérica, para el sector oeste de la Cordillera Volcánica Central (donde se ubica el área de estudio), se afirma lo siguiente:

"La evidencia histórica indica que el sismo máximo ocurrido en esta zona es de 6,5; sin embargo, considerando el tamaño de las fallas activas presentes, podría sugerirse magnitudes esperables cercanas a $\mathrm{Mw}$ 6,8" (Molina et al., 2008).

En relación con las aceleraciones máximas obtenidas, los valores calculados son consistentes con el estudio realizado por Ramírez et al. (1996) para el Valle Central de Costa Rica (donde se incluye el área de estudio), concretamente con el Mapa de Amenaza de Amplificación Sísmica de la Gran Área Metropolitana de Costa Rica, en el cual se estiman valores esperados entre $0,30 \mathrm{~g}$ y 0,70 g para el área de estudio. En la presente investigación se obtienen valores de aceleración entre $0,30 \mathrm{~g}$ y 0,76 $\mathrm{g}$ en el caso del sismo de diseño sobre la falla Ángel, y entre $0,36 \mathrm{~g}$ y $0,78 \mathrm{~g}$ para el epicentro asociado a la falla Sabanilla. A pesar de la proximidad de los valores cabe destacar que la escala del mapa de Ramírez et al. (1996) es 1:200.000 por lo que no contó con el detalle geológico para obtener valores más precisos- En esta investigación se utilizó cartografía geológica más reciente y detallada lo que resultó en valores puntuales de amplificación para depósitos de lahares y acumulaciones de tefras que sobrepasaron los $0,70 \mathrm{~g}$. 


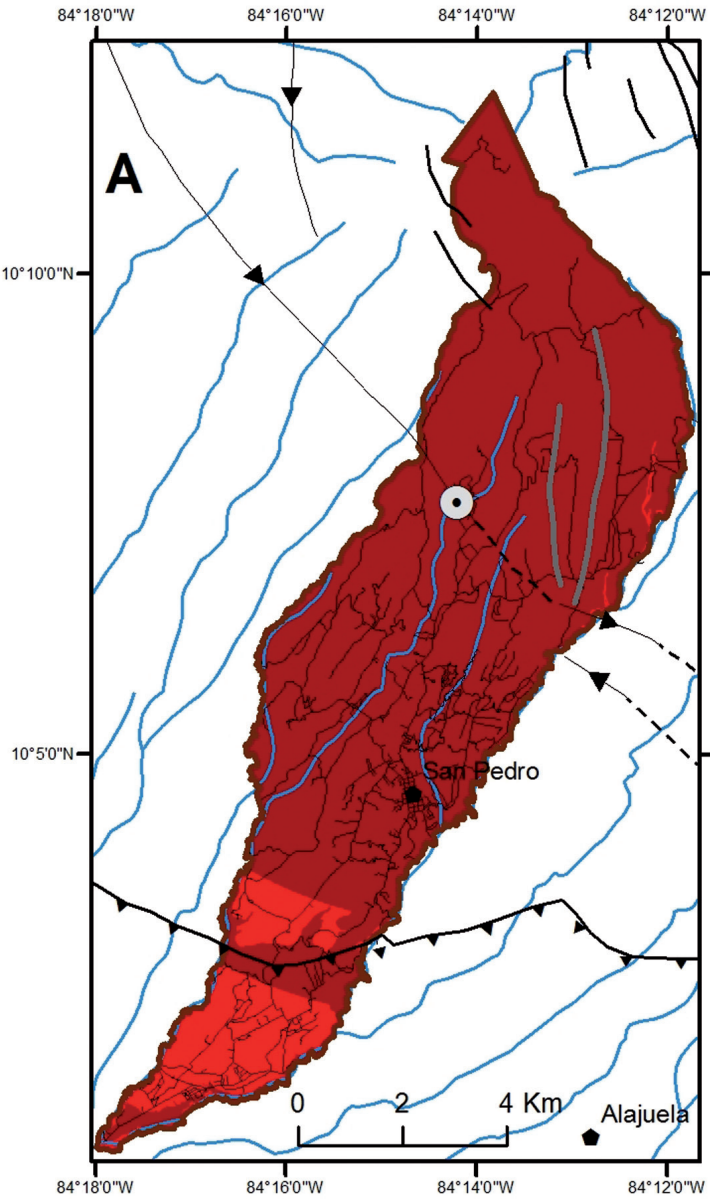

Simbología

Fallas

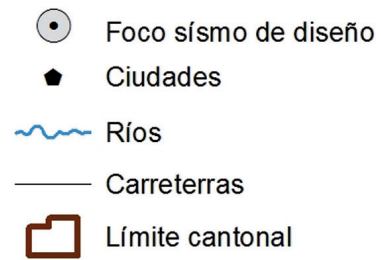

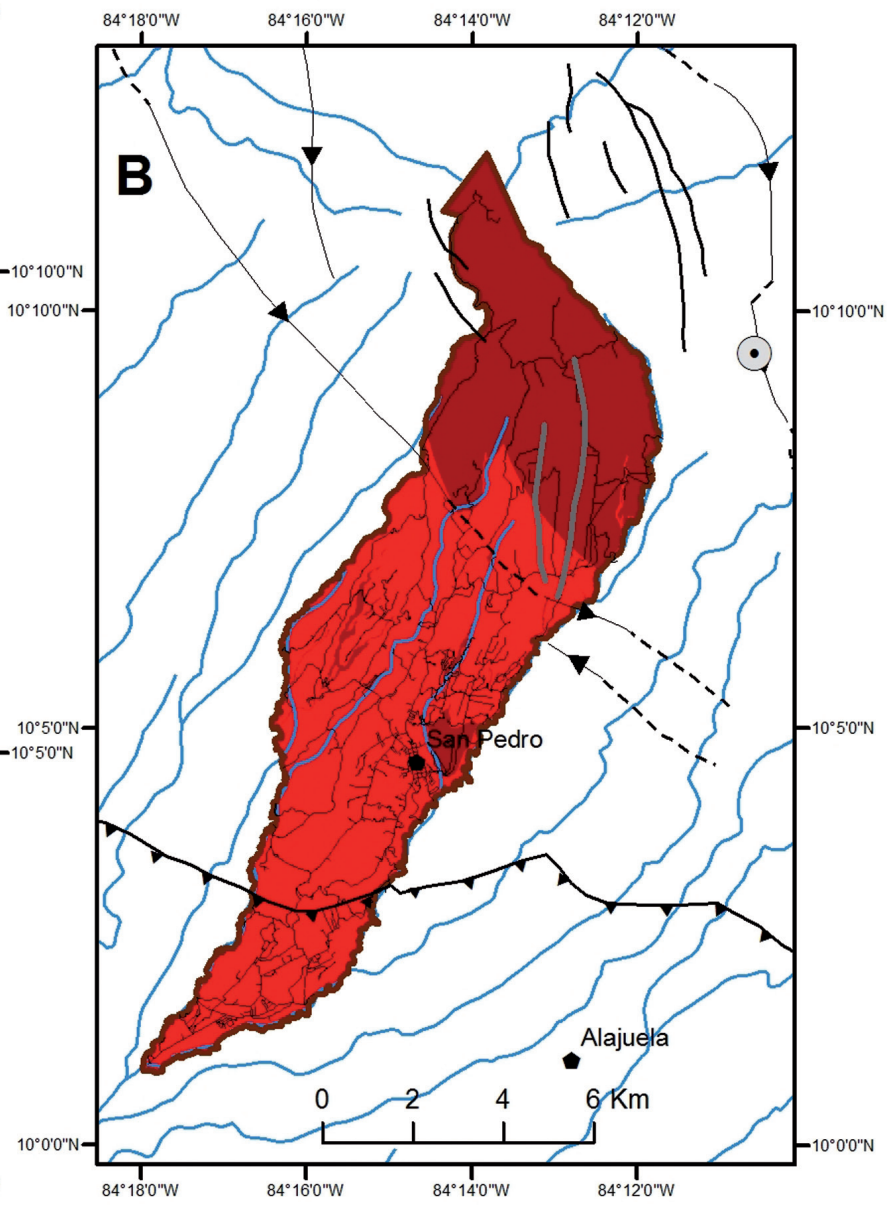

Distribución de Intensidades obtenidas para dos sismos de diseño

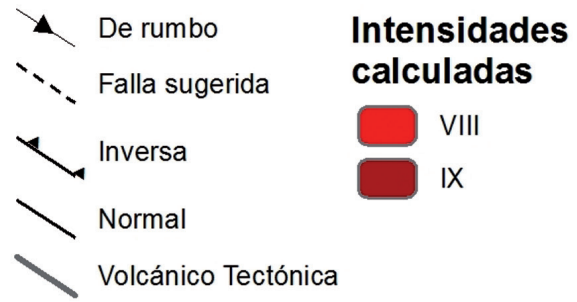

FIGURA 10. Escenarios de intensidad máxima esperadas a partir de sismos de diseño. A. sismo de diseño atribuido a la falla Sabanilla. B. sismo de diseño originado en la falla Ángel.

De acuerdo con el Código Sísmico de Costa Rica, el área de estudio se encuentra en la zona sísmica III y suelo tipo S3, donde es de esperar una aceleración pico efectiva de diseño de $0,36 \mathrm{~g}$ para un periodo de retorno de 475 años. Al transformar el valor medio de PGA obtenido para el sismo de diseño en la falla Sabanilla, siguiendo la recomendación del Laboratorio de Ingeniería Sísmica (2015), se obtiene un valor equivalente de $0,4 \mathrm{~g}$ que no difiere sustancialmente al referido en el Código Sísmico, no obstante, los valores debidos a la amplificación por efecto de sitio si exceden significativamente este favor. En ambos casos las diferencias pueden ser atribuidas a lo señalado por Climent et al. (2008) en el sentido de que la Ley de atenuación de Climent et al. (1994) sobreestima los valores de PGA en distancias menores a $200 \mathrm{~km}$. 


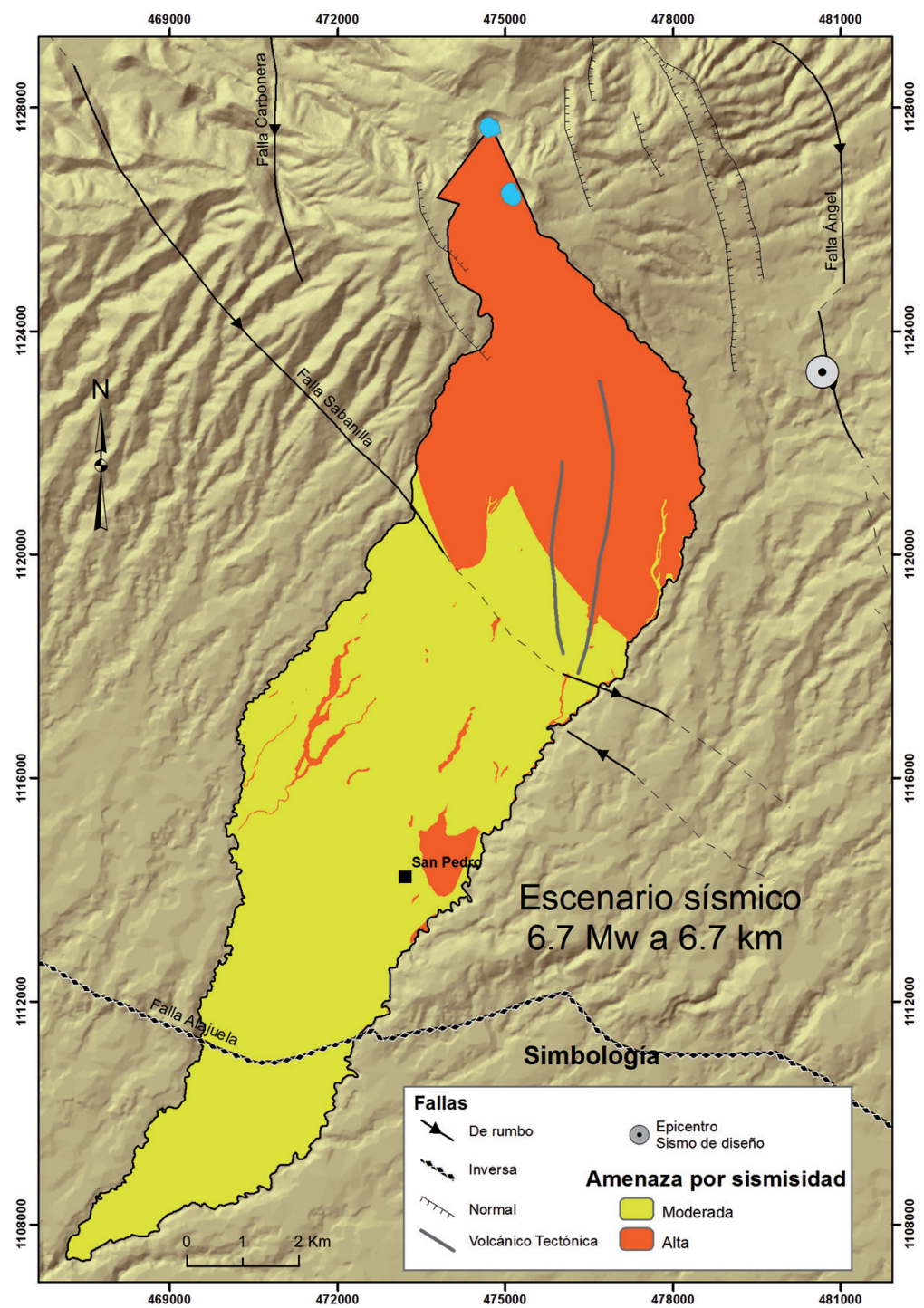

FIGURA 11. Indicador de amenaza sísmica acorde con la escala cualitativa utilizada en el Índice de Fragilidad Ambiental.

En el estudio de la amenaza sísmica en Costa Rica (Climent et al., 2008), para la zona de estudio se esperan aceleraciones entre 500 y 600 gales para un periodo de retorno de 500 años. Esta tendencia general es la que se obtuvo en esta investigación (FIGURA 9), no obstante, la escala de la información geológica permitió identificar posibles áreas de amplificación por efectos local que sobrepasan estos valores.

Por otra parte, el terremoto de Cinchona de magnitud $\mathrm{M}_{\mathrm{w}}$ 6,1 presentó una isosista máxima de IX con un diámetro máximo de alrededor de $8 \mathrm{~km}$ (FIGURA 7), por lo que cabría esperar que un sismo de diseño sobre la falla Ángel de magnitud $\mathrm{M}_{\mathrm{w}} 6,7$ a una profundidad similar presente una amplia zona con intensidad IX IMM, como se muestra en la FIGURA 10 B.
En relación con el OT, a partir de estos dos escenarios no se recomienda el uso del suelo urbano en la sección norte del cantón, dado que podría verse afectada por intensidades de entre VIII y IX IMM y que este sector se caracteriza por la presencia de fuertes pendientes y gruesas capas de regolito que podrían ser movilizadas como movimientos en masa durante un sismo fuerte, como fue descrito por Pittier para el terremoto de Fraijanes de 1888 (González, 1910) y por Barrantes et al. (2013) para el caso del terremoto de Cinchona de 2009. En especial debe evitarse la construcción de viviendas sobre depósitos de lahares que podrían amplificar las ondas sísmicas.

Estudios previos con enfoque probabilístico, como los realizados por Montero et al. (1997) y Climent et al. 
(2008), constituyen un valioso aporte a la evaluación de la amenaza símica en Costa Rica, no obstante su escala nacional hace pertinente el desarrollo de la metodología propuesta en este artículo debido a la consideración de las condiciones locales a nivel municipal y en particular como mejora al método de valoración de la amenaza sísmica establecido en el IFA, para su incorporación en el OT.

\section{CONCLUSIONES}

La zona de estudio ha sido afectada por varios terremotos entre los que se destacan el terremoto de Fraijanes de 1888 y el terremoto de Cinchona de 2009. La existencia de fallas próximas con potenciales sísmicos de $M_{w} 6,7$ justifica el presente estudio como un elemento básico en la toma de decisiones para el OT.

Con base en el uso de Sistemas de Información Geográfica, la disponibilidad de una base de datos sísmica y la recopilación de la información histórica, fue posible adaptar una metodología determinista a la estimación de la amenaza sísmica para su utilización en el OT, evitando el uso de aproximaciones basadas en cálculos simples, como los propuestos en la normativa IFA para Costa Rica.

De acuerdo con el peor escenario posible, se presenta una alta amenaza sísmica con potencial para generar intensidades de IX en la mayor parte del cantón. Esta información puede servir de base para la adopción de medidas de restricción en uso urbano del suelo, en especial en la sección norte del cantón de Poas y sobre los cañones de los ríos, donde las fuertes pendientes predisponen la ocurrencia de deslizamientos como los disparados por el terremoto de Cinchona en el 2009.

La ciudad de San Pedro, cabecera del cantón, se encuentra expuesta a intensidades de hasta IX IMM en caso del peor escenario, es por esta razón que se deben tornar medidas para reforzar la infraestructura crítica de la ciudad y asegurar un correcto cumplimiento del código sísmico, así como planificar cuidadosamente el emplazamiento de las zonas residenciales para evitar que se repita lo ocurrido en Cinchona.

\section{AGRADECIMIENTOS}

Esta investigación fue realizada en el Observatorio Sismológico y Geofísico del Suroccidente Colombiano (OSSO). Agradecemos al Observatorio Vulcanológico y Sismológico de Costa Rica (OVSICORI) por los datos sismológicos aportados para apoyar este trabajo.
Los autores agradecen las correcciones, comentarios y sugerencias del Dr. José Leonardo Álvarez Gómez del instituto INTER de Nicaragua y a los evaluadores anónimos de ésta y anteriores versiones, quienes han contribuido a mejorar sustancialmente el contenido y presentación de este trabajo.

\section{REFERENCIAS}

Abrahamson, N.A. 2006. Seismic hazard assessment: problems with current practice and future developments. In: first European conference on earthquake engineering and seismology, Geneva, Switzerland, pp. 3-8.

Alvarado, G., Morales, L.D., Montero, W., Climent, A., y Rojas, W. 1988. Aspectos sismológicos y morfotectónicos en el extremo occidental de la Cordillera Volcánica Central de Costa Rica. Revista Geológica de América Central, 9: 75-98.

Barquero, R. 2009. El terremoto de Cinchona del 8 de enero de 2009. RSN, San José, 138p.

Barrantes, G. 2012. Deficiencias del Índice de Fragilidad Ambiental en la valoración de las amenazas naturales para la planificación territorial. En torno a la prevención, pp. 1-9.

Barrantes, G., Jiménez, C., y Ocón, M.J. 2013. Deslizamientos provocados por el terremoto de Cinchona, Costa Rica. Revista Geográfia de América Central, 51: 89-100.

Benito, B., y Jiménez, E. 1999. Peligrosidad sísmica. Física de la Tierra, 11: 11-45.

Climent, A., Taylor, W., Ciudad Real, M., Strauch, W., Villagrán, M., Dahle, A., et al. 1994. Spectral strong motion attenuation in Central America. NORSAR Technical Report, 2-17, 46p.

Climent, A., Alvarado, G., y Boschini, I. 2006. PH Toro 3: estudio de amenaza sísmica. Instituto Costarricense de Electricidad, San José, pp. 22.

Climent, A., Rojas, W., Alvarado, G., y Benito, B. 2008. Proyecto Resis II: Evaluación de la amenaza sísmica en Costa Rica. RSN:UCR-ICE, Universidad de Madríd, $125 \mathrm{p}$.

Escuela Centroamericana de Geología. 2005. Geología del cantón de Poás y estudios adicionales. San José, Addendum Convenio AyA-FUNDEVI. 198p. 
González, C. 1910. Temblores, terremotos, inundaciones y erupciones volcánicas en Costa Rica. San José, Tipografía Avelino Alsina. 200p.

González, L., Ferrer, M., Ortuño, L., y Oteo, C. 2002. Ingeniería Geológica. Madrid, Pearson Educación. $744 \mathrm{p}$.

Herrera, B. 2011. Notas del curso geodinámica y problemas ambientales. Instrumentos de Gestión Ambiental . Chapingo, México, 120p.

INEC. 2012. INEC Costa Rica. Censo 2011 Resultados. Consultado el 15 de Octubre de 2012. http://www.inec. go.cr/Web/Home/GeneradorPagina.aspx

International Seismological Centre. 2014. Reference Event Bulletin. Consultado el 30 de Septiembre de 2014. http://www.isc.ac.uk

Laboratorio de Ingeniería Sísmica. 2015. Universidad de Costa Rica. Consultado el 22 de Julio de 2015. http:// www.lis.ucr.ac.cr/index.php?id=230

Laurent, J. 2009. Evaluación económica e las pérdidas y daños. En: Barquero, R., El terremoto de Cinchona, 8 de enero de 2009, RSN, San José, pp. 101-127.

Linkimer, L. 2008. Relationship between Peak Ground Acceleration and Mofified Mercalli Intensity in Costa Rica. Revista Geológica de América Central, 38: 81-94.

Massiris, A. 2005. Fundamentos conceptuales y metodológicos del ordenamiento territorial. Universidad Pedagógica y Tecnológica de Colómbia, 121p.

McGuire, R.K. 2001. Deterministic vs probabilistic earthquake hazards and risks. Soil Dynamics and Earthquake Engineering, 21(5): 377-384.

Molina, E., Marroquín, G., Escobar, J., Talavera, E., Rojas, W., Climent, A., Camacho, E., Benito, B., y Lindholm, C. 2008. Proyecto RESIS II: evaluación de la amenaza sísmica en Centroamérica. CEPREDENAC. 227 p.

Montero, W. 1989. Sismicidad Histórica de Costa Rica 1938 - 1910. Geofísica Internacional, 28 (3), 531-559.

Montero, W. 2001. Neotectónica de la región central de Costa Rica: frontera oeste de la microplaca de Panamá. Revista Geológica de América Central, 24: 29-56.
Montero, W., y Morales, L.D. 1988. Zonificación sísmica del Valle Central. Memoria del $4^{\circ}$ seminario de ingeniería estructural, pp. 335-351.

Montero, W., y Kruse, S. 2006. Neotectónica y geofísica de la falla Aguacaliente en los valles de Coris y el Guarco, Costa Rica. Revista Geológica de América Centra, 34-35: 43-58.

Montero, W., Rojas, W., Bochini, I., y Barquero, R. 1990. Neotectónica de la región de Puriscal: origen del enjambre sísmico de mayo-julio de 1990. RSN UCRICE, San José, 13p.

Montero, W., Peraldo, G., y Rojas, W. 1997. Proyecto de amenaza sísmica de América Central. IPHG. 79p.

Montero, W., Soto, G., Alvarado, G., y Rojas, W. 2010. División del deslizamiento tectónico y transtensión en el macizo del Volcán Poás (Costa Rica), basado en estudios neotectónicos y de sismicidad histórica. Revista Geológica de América Central, 43: 13-36.

Morales, L.D., Montero, W., y Madrigal, R. 1992. El terremoto de Fraijanes de 1888 y el sistema de fallas de Alajuela: implicaciones del peligro sísmico potencial para el Valle Central. Revista Geográfica de América Central, 25-26: 227-240.

Muñoz, D. 1989. Conceptos básicos en riesgo sísmico. Física de la Tierra, 1: 199-215.

OVSICORI. 2013. Base de datos sismográfica. Heredia, Costa Rica.

Poder ejecutivo. (4 de Mayo de 2006). Decreto $\mathrm{N}^{\circ}$ 32967-MINAE: Manual de instrumentos técnicos para el proceso de evaluación de impacto ambiental-PARTE III-. La Gaceta Diario Oficial , pp. 2-29.

Poder ejecutivo. (13 de julio de 2012). Decreto $\mathrm{N}^{\circ}$ 37070 “Código Sísmico de Costa Rica 2010”. La Gaceta Diario Oficlal, Alcance digital N ${ }^{\circ} 94$.

Ministerio de Vivienda, Ciudad y Territorio (19 de septiembre de 2014). Decreto 1807. República de Colombia. 19p.

PREDECAN. 2009. Incorporando la gestión del riesgo de desastres en la planificación y gestión territorial: guía técnica para la interpretación y aplicación del análisis de amenazas y riesgos. Secretaría General de la Comunidad Andina, Lima. 100p. 
Protti, M., González, V., Freymueller, J., and Doelger, S. 2012. Isla del Coco, on Cocos Plate, converges with Isla de San Andrés, on the Caribbean Plate, at $7,8 \mathrm{~mm} /$ yr. Revista Biología Tropical , 60 (3): 33-41.

Protti, M., González, V., Schwartz, S., Dixon, T., Newman, A., Feng, L., y Marshall, J. 2014. El terremoto de Nicoya $\left(\mathrm{M}_{\mathrm{w}}\right.$ 7.6), Costa Rica, del 5 de setiembre del 2012: un caso exitoso de anticipación. En torno a la prevención, pp. 6-12.

Pujadas, R., y Font, J. 1998. Ordenación y planificación territorial. Madrid: Editorial Síntesis S.A., 399p. LIBRO

Ramírez, R., Santana, G., y Chacón, O. 1996. Mapa de amplificación sismica del Valle Central, Costa Rica. Revista Geológica de América Central, 19-20: 37-55.

Rojas, W., Bungum, C., and Lindholm, C. D. 1993. Historical and recent earthquakes in Central America. Revista Geológica de América Central, 16: 5-21.

Ruegg, J. C. 1994. Deformación de la corteza terrestre y terremotos: aplicación al estudio del Ciclo Sísmico en el norte de Chile. Física de la Tierra, 6: 241-243.

Ruiz, P., Gazel, E., Alvarado, G., Carr, M. J., y Soto, G. 2010. Caracterización geoquímica y petrográfica de las unidades geológicas del macizo del volcán Poás, Costa Rica. Revista Geológica de América Central, 43: 37-66.

Saborío, J., Saborío, M., y Mora, S. 2014. Gestión municipal del riesgo de desastres: normas y elementos básicos para su inclusión en el ordenamiento territorial. CNE, San José. 48p.

Schmidt, V., Dahle, A., and Bungum, H. 1997. Costa Rican spectral strong motion attenuation, NORSAR Technical Report, 45p.

Smith, K., and Petley, D. N. 2007. Environmental hazards. New York: Routledge. 383p.

Subsecretaría de Desarrollo Regional y Administrativo, Gobierno de Chile. 2011. Guía análisis de riesgos naturales para el ordenamiento territorial. CEPAL, Santiago. $144 \mathrm{p}$.

Trifunac, M. D., and Brady, A. G. 1975. On the correlation of seismic intensity scales with the peaks of recorded ground motion. Bulletin of the Seismological Society of America, 65: 139-162.
Wells, D., and Coppersmith, K. 1994. New empirical relationships among magnitude, rupture length, rupture width, rupture area, and surface displacement. Bulletin of the Seismological Society of America, 84(4): 9741002 .

Westen, C.V., Montoya, L., Boerboom, L., y Vargas, R. 2012. Aplicación de SIG para la evaluación de la amenaza sísmica y el riesgo asociado: Kathmandu, Nepal. Consultado el 1 de Junio de 2012. www.zotero. org/groups/research_icg/items/6AZNTTUB

Zhao, J.X.; Zhang, J., Asano, A., Ohno, Y., Oouchi, T., Takahashi, T., Ogawa, H., Irikura, K., Thio, H.K., and Somerville, P.G. 2006. Attenuation Relations of Strong Ground Motion in Japan Using Site Classification Based on Predominant Period. Bulletin of the Seismological Society of America, 96: 898-913.

Trabajo recibido: agosto 10 de 2015

Trabajo aceptado: mayo 26 de 2016

Manuscrito publicado en internet: junio 28 de 2016 